\title{
Prescriber Opioid Patterns Following Cesarean Section Pre and Post Intervention at a Large Academic Institution: a retrospective cohort study.
}

\author{
Alexa Cohen ${ }^{1}$, Talitha Bruney ${ }^{1}$, Melissa Fazzari ${ }^{2}$, and Gayatri Nangia ${ }^{2}$ \\ ${ }^{1}$ Montefiore Hospital and Medical Center \\ ${ }^{2}$ Albert Einstein School
}

June 24, 2020

\begin{abstract}
Objective: To describe opioid prescribing patterns following cesarean section before and after the New York State Department of Health (NYSDOH) mandated the Opioid Prescriber Training Program. Design: This is a retrospective cohort study. Setting: A single hospital institution in New York City, United States. Sample: 1,494 women hospitalized for cesarean section between July 2016 and August 2018. Methods: Chart review of patient records. Main Outcome Measures: Primary outcome was total amount of narcotic prescribed before and after the Opioid Prescriber Training. Secondary outcomes included outpatient opioid prescription habits by provider level, as well as outpatient opioid prescription patterns related to the amount of inpatient narcotic use, and patient, surgical, and hospital-specific factors. Results: There was a significant difference in narcotics prescribed before and after the training. The median dose of narcotic prescribed pre and post intervention was 150 morphine milligram equivalents (MME) which is equal to 20 pills of $5 \mathrm{mg}$ of oxycodone. Pre-training, $41.1 \%$ of prescriptions amounted to $>150 \mathrm{MME}$, compared with $21.3 \%$ post-training ( $\mathrm{p}$-value for association $<0.001$ ). Post-training, all provider levels had reduced narcotic prescriptions in the category of >150 MME. Neither inpatient opioid use, patient demographic, surgical nor hospital factors affected opioid prescriber patterns. Conclusion: This suggests the NYSDOH mandated opioid training course had an effect on opioid prescribing patterns with the greatest impact noted in residents. Funding: None. Keywords: discharge prescriptions, obstetric narcotics, opioid training, post-cesarean narcotics.
\end{abstract}

\section{Tweetable Abstract}

A retrospective cohort study of provider prescribing patterns of narcotic after a cesarean both pre and post a mandated opioid training course showed a decrease in the mount of narcotic prescribed.

\section{Main Text}

\section{Introduction:}

Opioid abuse in the United States has heightened to dangerous proportions in the past 15 years. According to the Centers for Disease Control and Prevention (CDC), the opioid-related death toll in the United States (US) increased five-fold from 1999 to 2016. ${ }^{1}$ As a result in 2017 the US Department of Human and Health Services called this "Opioid Crisis" a state of public emergency, and subsequently is funding research and issuing strategies to combat this ongoing problem.

A major contributing factor to this epidemic is the increase in opioid prescriptions. Per the CDC, from 1999 through 2014 opioid prescription sales increased four-fold, and of the 42,000-plus opioid related deaths in 2016, $40 \%$ were attributed to a prescription opioid. ${ }^{1}$ This rise in opioid prescriptions increases narcotic availability as well as potential for abuse and misuse. A 2014 national survey in the US quoted 10.3 million 
people taking prescription opioids for nonmedical reasons, commonly using opioids not prescribed to them or using their prescription for other indications than were initially prescribed. ${ }^{2}$ In addition, there is a correlation between prescription opioid use and future heroin abuse. ${ }^{3}$

In response to the opioid crisis in New York State, the New York State Department of Health (NYSDOH) mandated that all providers who prescribe narcotics, including residents under a faculty Drug Enforcement Administration (DEA), complete a three-hour online course entitled "Opioid Prescriber Training Program" by July 1, 2017. This course describes the various options for pain management in the outpatient and palliative care setting, the physiology of narcotics, evidence regarding opioid overuse and consequences of addiction. Most states have specific required coursework, lasting 1-3 hours, for opioid prescribers in this vane. However, no other state mandates residents prescribing narcotics to complete a mandatory online training course.

Cesarean section is the most common surgical procedure undergone by reproductive aged women. According to the CDC, there were 1,258,581 Cesarean Deliveries in the US in 2016. ${ }^{1}$ At our institution, Montefiore Medical Center, Weiler Campus there are approximately 4000 deliveries per year and our Cesarean Delivery rate is about $30 \%$. According to a nationwide survey, $85 \%$ of women receive a prescription for opioids after their cesarean section. ${ }^{4}$ At our institution, Montefiore Medical Center, all patients undergoing cesarean section receive a prescription for opioids upon discharge unless there is a medical contraindication and/or patient declines.

Data regarding opioid prescriptions after cesarean section has revealed that for the most part, the amount of narcotics prescribed surpasses the amount consumed. ${ }^{5,6}$ One prospective survey study showed only half the amount of prescribed narcotic tablets was reportedly consumed by patients. ${ }^{5}$ However much of this data is prior to 2017, and there is an inadequate amount of knowledge on obstetrician prescriber habits after the NYSDOH mandated that all prescribers take an opioid course.

Our primary objective was to describe opioid prescriber practices at time of hospital discharge following cesarean section in a large cohort of women before and after the NYSDOH mandated the Opioid Prescriber Training Program. Secondary aims were to analyze opioid prescription habits by provider level as well as to identify trends in opioid prescription patterns related to the amount of inpatient opioid use as well as surgical and/or hospital specific factors.

\section{Methods:}

This is a retrospective cohort analysis of women at a single high-volume academic institution who underwent a cesarean section and were discharged from the hospital. This study included 1,494 women hospitalized for cesarean delivery from July 1, 2016 to December 31, 2016 and then from January 1, 2018 to August 31, 2018, excluding women within the one year surrounding the deadline for the NYSDOH mandated Opioid Prescriber Training Program (January 1, 2017 to December 31, 2017). This tertiary center is in an urban area with a multilingual, racially and ethnically diverse population. Over 80 attending physicians, residents, and physician assistants employed by the hospital system treated these patients.

At our institution, women routinely receive neuraxial anesthesia (epidural and/or spinal) for cesarean delivery. In the post-operative period, the obstetrical team manages pain, and women generally receive multimodal pain management including long acting narcotics, most commonly oxycodone or Percocet (oxycodoneacetaminophen). Discharge medications are prescribed at the individual provider's discretion. There are no current guidelines at our hospital for inpatient or outpatient narcotic prescriptions. Though there is a postCesarean order set in our electronic medical record system EPIC, there is no discharge navigator or discharge order set that includes prescriptions.

The Institutional Review Board at Montefiore Medical Center, Albert Einstein College of Medicine approved this study. We obtained information via chart review within EPIC. Our center's electronic medical record system includes all outpatient and inpatient records. We queried demographic information including age, race, ethnicity and primary language. We then obtained all clinical and pharmacologic data, including 
patient and surgery specific characteristic as well as inpatient medications and outpatient prescriptions, directly from the electronic medical record. The data was transferred to an electronic database and double checked independently by two members of the research team. If there was missing data, it was labeled as "unknown". We used the same electronic record to see if a narcotic was prescribed at patient discharge, and if so, we included information on the type, strength and number of narcotic pills prescribed. We converted all narcotics into total morphine milligram equivalents (MME) using conversion rates from CDC.gov to more effectively compare amounts among the different opioids. ${ }^{7}$ We obtained this value by converting each opioid dosage to MME and then multiplying by the number of pills. ${ }^{8}$ The literature frequently uses this MME conversion to compare amounts between narcotics. Our primary outcome was total MME prescribed for outpatient use. We analyzed the total outpatient MME prescribed at discharge before and after the mandated NYSDOH Opioid Prescriber Training. Secondary outcomes included analyzing outpatient opioid prescription habits by provider level as well as identifying trends in outpatient opioid prescription patterns related to the amount of inpatient narcotic use, and patient, surgical and hospital-specific factors. Since this study was not focused on actual patient use, we did not collect information on whether the prescriptions were filled or not.

We computed descriptive statistics (frequencies, medians and interquartile ranges) to summarize patient, surgical and hospital-specific factors pre and post intervention, as well as across all patients. We assessed the association between cohort (pre vs. post-intervention) and each factor via chi-square test. In-house opioid use was categorized as $<50,50-100$, and $>100$ MME. Total amount of opioid prescribed at discharge was categorized as $0,<150,150$, and $>150 \mathrm{MME}$. These categories were chosen for the discharge prescriptions since $150 \mathrm{MME}$ was the median amount of narcotic prescribed both pre and post-intervention. In order to examine the association between opioid prescription and patient, surgical and hospital factors, ordinal logistic regression models were estimated for both the pre and post-intervention periods. We examined univariate and fully adjusted models based on a set of a prioriclinically relevant variables. Age was categorized as [?]25 years, 26-30, 31-35,36-40,41-45,46-50, and > 50. BMI was categorized as normal $(<25)$, overweight (25-29.99), obese 1 (30-34.99), obese $2(35-39.99)$ and obese $3(40+)$. Surgical time was categorized as $<$ 30 minutes to $>180$ minutes in 30-minute increments. Age, surgical time, and BMI were entered into the model as ordinal variables based on the above categorization. Odds ratios (OR) and corresponding $95 \%$ confidence limits were estimated. Two-sided p-values less than 0.05 were considered statistically significant. All analyses were performed in SAS version 9.4 (SAS Institute Inc., Cary NC).

\section{Results:}

\section{Demographics}

Our study included 1,494 patients who received a Cesarean Section at Montefiore Medical Center Weiler Campus between July 1, 2016 to December 31, 2016 and January 1, 2018 to August 31, 2018 and were discharged from the hospital. Of these patients, 739 had their cesarean between July 1, 2016 through December 31, 2016, before the mandated NYDOH opioid training (the "pre-intervention" group), and 755 had their cesarean between January 1, 2018 through August 31, 2018, after the mandated opioid training (the "post-intervention" group). The patient demographic and obstetric characteristics were similar between groups (Table 1a). The majority of patients were less than 35 years old, Hispanic, English-speaking and nulliparous. Most patients were overweight or obese (BMI $>25 ; 77.8 \%$ ). In addition, more than half of the patients in each group did not have any prior cesarean sections $(51.3 \%)$

The patients' hospital and surgical characteristics were similar between groups. Table $1 \mathrm{~b}$ shows the relevant details about each cesarean section and hospital course stratified by whether the patients underwent the surgery before or after the mandated opioid training. The majority of patients in both groups had unscheduled cesarean sections (pre-intervention: $67.3 \%$, post-intervention: 62.8\%). The most common indication for cesarean section was repeat procedure followed by fetal intolerance, and labor dystocia. In majority of the cases the estimated blood loss was less than 1000cc. However, there was a statistical significance between cohorts with $71 \%$ of cases pre-intervention reporting a blood loss less than 1000 cc versus $60.7 \%$ post-intervention $(\mathrm{p}=0.001)$. In most cases sutures were used for skin closure, but the percentage of surg- 
eries utilizing staples significantly decreased from $9.3 \%$ pre-intervention to $5.4 \%$ post-intervention (p-value $=0.009$ ). Surgical time varied significantly as well. The majority of cases in the pre-intervention group lasted between 30 to 60 minutes versus between 60 to 90 minutes in the post-intervention group (p-value $=0.005)$. For both groups, the most common length of stay in the hospital was 2-3 days. Many of the patients in both groups had horizontal skin incisions (97\%) and neuraxial anesthesia (97-98\%). Most had no other procedures performed at the time of cesarean (86-88\%), or in-hospital infections (89-90\%). Finally, there was a statistically significant association between the level of provider prescribing the narcotics and cohort (pre vs post intervention) $(\mathrm{p}$-value $=0.001)$. However, within both the pre and post intervention groups, the largest percentage of narcotic prescribers were first year residents (pre-intervention: 66.2\%, post-intervention: $72.5 \%$ ).

\section{Outcomes}

Table 2 summarizes the prescribing patterns pre and post training. A lower percentage of prescriptions posttraining were $>150 \mathrm{MME}(21.3 \%)$ as opposed to prior to the training $(45.1 \%)$ and a higher percent were $<$ 150 MME post-training (22.5\% vs. $13.7 \%$; p-value for association $<0.001)$. Despite this shift, the median amount prescribed remained $150 \mathrm{MME}$ pre and post-intervention. Table 3 examines prescribing patterns stratified by prescribing provider level. Pre-intervention, there was a statistically significant association between provider level and narcotic prescription category $(\mathrm{p}$-value $=0.03)$. Residents were 1.63 times more likely to prescribe higher categories of narcotics compared to physician assistants, fellows, and attendings (95\% CI: $(1.04,2.54))$. In an a priorimodel adjusted for age, race, body mass index (BMI), surgery time, prior cesarean section, in-house infection, in-house narcotic use and whether other procedures were performed, this association remained statistically significant $(\mathrm{OR}=3.42 ; 95 \%$ CI: $(1.14,10.24)$; data not shown). Posttraining, all provider levels had reduced proportions of narcotic prescriptions in the $>150 \mathrm{MME}$ category. We observed large reductions in narcotic prescriptions $>150$ MME after the training period in $2^{\text {nd }}$ through $4^{\text {th }}$ year residents such that during the pre-intervention phase, $84 \%$ and $65 \%$ of $2^{\text {nd }}$ and $3-4^{\text {th }}$ year residents prescribed $>150$ MME respectively, compared to $22 \%$ and $28 \%$ post-intervention. In post-intervention univariate and adjusted models, there were no statistically significant associations between provider level and the amount category of narcotics prescribed (p-values 0.30 and 0.65 respectively). Total in-house narcotic use was observed to be associated with higher levels of narcotics prescribed post-intervention in univariate analysis, however this effect was not statistically significant in the a priori adjusted model (OR $>100$ vs. [?] $50 M M E=1.10,95 \%$ CI: $(0.71,1.72))$. There were no patient or surgical factors that were associated with the amount of narcotics prescribed in either univariate or multivariable analysis. Therefore, with the exception of in-house narcotic use, multivariable models did not yield qualitatively different results and were thus not included. Table 4 summarizes univariate associations for selected patient and surgical characteristics pre and post intervention.

\section{Discussion:}

\section{Main Findings}

In our retrospective sample of women undergoing cesarean section, opioid prescription patterns altered following the NYSDOH mandated opioid prescriber training course. After this intervention, we found a statistically significant shift towards a lower amount of narcotic prescribed. Our data also suggests that amount of opioid prescribed did not correlate with opioid consumption in the hospital, patient demographic factors (i.e. BMI, age, race), surgical factors (surgery length, indication for cesarean, estimated blood loss, skin incision, closure, anesthesia), and hospital factors (length of stay, infection).

Cesarean section is the most common surgery undergone by women. There is a paucity of data on opioid prescription patterns and normative opioid consumption after cesarean delivery, which poses a challenge for obstetricians who are attempting to prescribe an appropriate amount of narcotic for pain control. In light of the opioid epidemic, there have been statewide and national attempts towards decreasing narcotic use. Our study demonstrated that the provider training course mandated by the NYSDOH altered prescriber practices after January 1, 2018 at our institution. 
The median amount of prescribed narcotic was $150 \mathrm{MME}$ both pre and post-intervention because the most common prescription in both groups was 20 pills of $5 \mathrm{mg}$ oxycodone or Percocet which converts into 150 MME. Though 150 MME remained the median amount of narcotic prescribed, we observed a decline in the percentage of prescriptions $>150 \mathrm{MME}(21.3 \%)$ post-training as opposed to prior to training, a reduction that was particularly apparent in residents.

This apparent decline of prescriptions > 150 MME especially in residents is important because at many academic institutions, residents are primarily responsible for prescribing a large number of narcotics following all procedures. Although many states now require an opioid training course for providers prior to allowing prescriptions, New York is the only state, which mandates residents take a narcotic training course. The positive shift in our institution prescribing patterns after the mandated opioid training course helps support the argument that residents be mandated to take a course and have opioid training/education be included as part of the residency curriculum. This is especially significant since residents will move on to become prescribing Attendings themselves

\section{Strengths}

The main strength of our study is that we reviewed an extensive cohort of women in the pre and post intervention groups and we were able to look at prescriber patterns for a large number of diverse providers of varying levels using a reliable EMR system. This, therefore, provides an accurate picture of what is occurring at our facility.

\section{Limitations}

It is important to note that our study has some limitations. It is a retrospective study with a high risk, urban patient population, which may not be generalizable of all cesarean deliveries in the United States other countries. In addition, there was a significant difference in BMI and race between the groups. We can explain this by the large percentage of "unknown" race and BMI. We gathered the information about race and BMI from EPIC chart review, and usually secretaries enter in these demographics. We do not feel that our patient population changed after the intervention. In addition, between the two groups there was a significant difference in surgery time, estimated blood loss and skin closure. Surgeries were longer postintervention. We speculate that this was due to an increased number of newer and younger hires on faculty. Frequently junior attendings have longer surgical times when compared to more experienced faculty. The difference in estimated blood loss between groups may be explained by the implementation of "Quantitative Blood Loss" measurements where nurses calculated blood loss for deliveries using a standard formula. At our institution we noted that quantitative blood loss measurements tended to be higher than the providers estimated blood loss. Though we used estimated blood loss in our study, we understand the measurement of quantitative blood loss may bias providers' estimations as well. The majority of cases were closed using sutures. Though the decrease in the use of staples post-intervention was significant, we do not believe that this is clinically relevant, and would have to perform future studies on narcotic use with different methods of skin closure to determine a true association.

Our study only looked at our provides' prescription habits, and this may not accurately depict which prescriptions were filled by the patients and/or amount of narcotic consumed. There were a variable number of providers in each level, with the largest number of prescriptions being written by residents. Our total analysis did not account for prescription habits between providers of different levels. However, we do not feel that controlling for provider level would alter the effect since in general PGY1-2 in the pre-intervention group were PGY 3-4 in the postintervention group. Another limitation is that our institution piloted an Enhanced Recovery After Surgery or ERAS protocol around the same time-period that we performed our chart review. This trial included 58 patients and used an inpatient only post-operative EPIC order set. Data from the protocol demonstrated that ERAS was not associated with a reduction in postoperative narcotic use. ${ }^{9}$ Though there is a possibility the pilot may have affected our results post intervention, it is unlikely to be relevant since as mentioned above, a priori adjusted model showed no statistical significance between in house narcotic use and prescription doses. 


\section{Conclusion}

Our study suggests that the mandated opioid training course had an effect on prescribing patterns after cesarean section. In addition, we interestingly noted that neither amount of opioid consumed in the hospital, nor patient demographic, surgical or hospital factors played a part in influencing narcotic prescriptions. This is important as one would expect the amount of narcotic use inhouse to predict future use at home in the immediate post-operative setting, and therefore should be factored in when writing these prescriptions.

Next steps would include a prospective study looking at the percentage of patients that filled their opioid prescriptions and actual patient opioid consumption upon discharge. Although we demonstrated that prescription patterns at our hospital were not correlated with inpatient narcotic use, patient demographics or surgery characteristics, it is possible that outpatient opioid use may be associated with these characteristics. A future project would include developing an algorithm to combine relevant patient characteristics or surgery factors with actual patient use at home in order to guide providers in writing appropriate narcotic prescriptions upon discharge.

The difference in amount of narcotic prescribed after the training for patients post cesarean section may indicate a shift in provider attitudes surrounding opioids before and after the mandated New York State opioid training. However, it is important to note that this shift may be a result of the intervention or simply to a general increase in awareness about the opioid crisis during this time through the media and news. Our study addresses the validity of this mandated opioid training course as a means for altering provider narcotic prescribing habits. Our study is relevant outside of New York, as most other states require a similar opioid prescriber training program prior to allowing providers to send narcotic prescriptions.

\section{Disclosure of Interests:}

The authors declare that they have no conflicts of interest and nothing to disclose.

\section{Contribution to Authorship:}

T B was the primary investigator on this project. She was involved in initial conceptualization and project planning. She also finalized IRB protocol for approval. She supervised the initiation and progress of the entire project. She played a major role in revising final paper and was responsible for final review and editing.AC was involved in conceptualization of project with primary investigator. She wrote initial IRB protocol for approval, carried out the research, chart review, and recording of data into database. She played a large role in writing the original draft and in subsequent revisions. MF was the statistician on the project. She provided formal statistical analysis and helped interpret data findings and write methods and results section of paper. GN assisted in completing chart review and recording in the database and as well as in writing original draft.

\section{Details of ethics approval:}

The Institutional Review Board (IRB) at Montefiore Medical Center, Albert Einstein College of Medicine approved our research study on July $15^{\text {th }} 2019$.

\section{Funding:}

No funding was required for this study

\section{References:}

1. Martin JA, Hamilton BE, Osterman MJK, Driscoll AK, Drake P. Births: Final data for 2016. National Vital Statistics Reports; vol 67 no 1. Hyattsville, MD: National Center for Health Statistics. 2018.

2. Center for Behavioral Health Statistics and Quality. 2014 National Survey on Drug Use and Health: detailed tables. Rockville, MD: Substance Abuse and Mental Health Services Administration, 2015.

3. Compton WM, Jones CM, Baldwin GT. Relationship between nonmedical prescription-opioid use and heroin use. New Engl J Med. 2016;374:154-163. 
4. Prabhu M, Dubois H, James K, et al. Implementation of a Quality Improvement Initiative to Decrease Opioid Prescribing After Cesarean Delivery. Obstet Gynecol . 2018;132(3):631-636. doi:10.1097/AOG.0000000000002789

5. Bateman BT, Cole NM, Maeda A, Burns SM, Houle TT, Huybrechts KF, et al. Patterns of opioid prescription and use after cesarean delivery. Obstet Gynecol 2017;130:29-35.

6. Osmundson SS, Schornack LA, Grasch JL, Zuckerwise LC, Young JL, Richardson MG. Postdischarge Opioid Use After Cesarean Delivery. Obstet Gynecol. 2017;130(1):36-41.

7. https://www.cdc.gov/drugoverdose/pdf/calculating_total_daily_dose-a.pdf

[Accessed 5 Mar. 2020].

8. Badreldin N, Grobman WA, Chang KT, Yee LM. Opioid prescribing patterns among postpartum women. Am J Obstet Gynecol . 2018;219(1):103.e1-103.e8. doi:10.1016/j.ajog.2018.04.003

9. Teigen NC, Sahasrabudhe N, Doulaveris G, et al. Enhanced recovery after surgery at cesarean delivery to reduce postoperative length of stay: a randomized controlled trial. Am J Obstet Gynecol . 2020 Apr;222(4):372.

10. Hill, M.V., McMahon, M.L., Stucke, R.S., and Barth, R.J. Wide variation and excessive dosage of opioid prescriptions for common general surgical procedures. Ann Surg. 2017; 265: 709-714

11. Bates C, Laciak R, Southwick A, et al. Overprescription of postoperative narcotics: A look at postoperative pain medication delivery, consumption and disposal in urologic practice. J Urol. 2011;185:551 555.

12. As-Sanie S, Till SR, Mowers EL, et al. Opioid prescribing patterns, patient use, and postoperative pain after hysterectomy for benign indications. [Published online ahead of print November 3, 2017]. Obstet Gynecol. doi:10.1097/AOG.0000000000002344.

13. Holland E1, Bateman BT, Cole N, et al. Evaluation of a Quality Improvement Intervention That Eliminated Routine Use of Opioids After Cesarean Delivery. Obstet Gynecol. 2019 Jan;133(1):91-97

14. Dowell D, Haegerich TM, Chou R. CDC Guideline for Prescribing Opioids for Chronic Pain - United States, 2016. MMWR Recomm Rep 2016; 65:1.

15. Cuomo, A., Zucker, H. and Dreslin, S. (2017). Mandatory Prescriber Education Guidance. [online] Health.ny.gov. Available at: https://www.health.ny.gov/professionals/narcotic/docs/mandatory_education_guidance.pdf [Accessed 5 Mar. 2020].

16 Cuomo, A., Zucker, H. and Dreslin, S. (2017). Mandatory Prescriber Education Guidance. [online] Health.ny.gov. Available at: https://www.health.ny.gov/professionals/narcotic/docs/mandatory_education_guidance.pdf [Accessed 5 Mar. 2020].

\section{Hosted file}

Tables . docx available at https://authorea.com/users/336326/articles/462051-prescriber-opioidpatterns-following-cesarean-section-pre-and-post-intervention-at-a-large-academicinstitution-a-retrospective-cohort-study 